\title{
Research Progress of Locally Resonance Acoustic Metamaterials
}

\author{
Du Zhehua $^{1}$ \\ ${ }^{1}$ Wuhan Second Ship Design and Research Institute, 430205 Wuhan, China
}

\begin{abstract}
Bragg scattering phonon crystal and locally resonant acoustic metamaterials were introduced. In order to generate noise reduction, the lattice constant of Bragg scattering phonon crystal should be of the same order of magnitude as the wave length of the sound wave, therefore, its application field is limited. Locally resonant acoustic metamaterials consume sound energy by coupling its own resonant frequencies with those of sound waves at close range. Its size is two orders of magnitude smaller than the wavelength of sound wave; thus, the control of low-frequency noise by small-size acoustic metamaterials is realized. Locally resonant acoustic metamaterials have some extraordinary physical characteristic in the conventional medium for their special acoustic structural units, such as negative refraction and negative mass density. Especially in low frequency band, they have acoustic forbidden band in which the sound wave transmission is prohibited. Acoustic structural unit having resonant characteristics has been developed. Surface-mounted resonant element plate structures and thin film acoustic metamaterials are the normal types of locally resonant acoustic metamaterials. Their research and development provide a new method for low-frequency noise control.
\end{abstract}

\section{Introduction}

Acoustic metamaterials originated from Bragg scattering phonon crystals, which are new functional materials formed by periodic introduction of elastic solids in solid or fluid media. When sound wave in a certain frequency range propagates in the periodic elastic composite medium, a sound band gap similar to photon band gap will be generated, thus preventing sound wave propagation in the frequency range. Generally, only when the lattice constant of Bragg scattering type phononic crystal is in the same order of magnitude as the wavelength of acoustic wave that produces forbidden band, acoustic forbidden band can be produced. This limits the application range of Bragg scattering phononic crystals.

Traditional noise reduction materials or structures have excellent sound absorption or insulation effect on the medium and high frequency noise produced during equipment operation, but their suppression effect on the low frequency noise is poor. At the same time, as equipment has stricter requirements on the quality and thickness of noise reduction materials, the limitations of traditional noise reduction materials have become increasingly prominent.

In 2000, Liu proposed locally resonant phonon crystal [1-2]. Its structure is locally resonance structure formed by fixing hard core ball on the elastic film through the coating layer. When the frequency of sound wave in the medium is close to the resonance frequency of resonance unit, resonance unit and sound wave have strong coupling effect and consume sound energy. Compared with Bragg scattering phonon crystal which produces the same bandgap characteristics, the size is reduced by two orders of magnitude. Thus, the smallsize acoustic metamaterials can control long sound waves.

With the development of acoustic metamaterials, locally resonant acoustic metamaterials have been developed, such as acoustic structural metamaterials with resonant characteristics, structural acoustic metamaterials with surface attached locally resonance unit plate, and thin film acoustic metamaterials

\section{Acoustic structural metamaterials with resonance characteristics}

Acoustic structural metamaterials with resonance characteristics are the initial manifestations of locally resonance phononic crystals. It is an extension of the traditional Bragg scattering phononic crystal concept. Figure 1 shows the prototype of an acoustic structure metamaterial with resonance characteristics designed by Z. Liu et al. Its structure is a metal sphere with a diameter of $5 \mathrm{~mm}$, coated with silica gel layer, and then introduced into resin matrix to form a simple cubic lattice configuration. Its lattice constant is $1.55 \mathrm{~cm} \mathrm{[3].}$ Through theoretical calculation and experimental analysis, the phononic crystal has an acoustic band gap at $380 \mathrm{~Hz}$ and $1340 \mathrm{~Hz}$. This band gap has good sound insulation performance. Through the simulation analysis

\footnotetext{
*Corresponding author: shunli878@163.com
} 
of Comsol Multiphysics, it is found that this type of phonon crystal has negative effective dynamic mass density at this frequency, which produces the acoustic band gap.

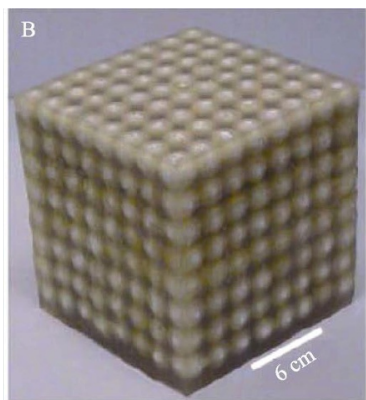

Fig. 1. Photographs of acoustic metamaterial prototype

Ding et al. buried open hollow sphere into sponge body and prepared a kind of acoustic metamaterial with negative elastic modulus [4], as shown in Figure 2. Through simulation calculation and experimental analysis, it is found that this type of material has excellent sound insulation performance between $900 \mathrm{~Hz}$ and $1500 \mathrm{~Hz}$.

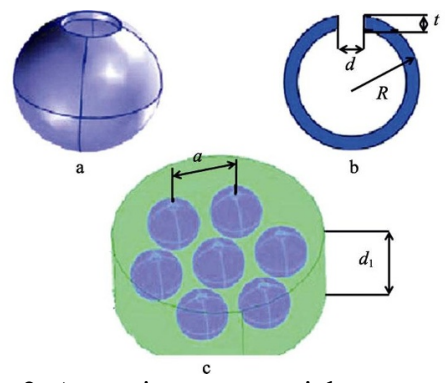

Fig. 2. Acoustic metamaterial prototype

\section{Acoustic metamaterials with surface- attached locally resonance unit plate structure}

There are a large number of plate structures in various types of equipment. Under acoustic or vibration excitation, when resonant frequency is consistent with or close to excitation frequency, plate structure will produce resonance and radiate acoustic waves to the outer space of plate. This acoustic radiation band is usually in the low frequency band. Xiao et al. used the principle of dynamic vibration absorber to form acoustic metamaterials by periodically introducing locally resonant units with resonant characteristics on the plate surface. When natural frequency of resonance unit is in the mass density control area where plate structure produces sound transmission, the sound insulation volume of the plate structure in the mass control area can be effectively improved [5]. Figures $3 \mathrm{a}$ and $3 \mathrm{~b}$ show the acoustic structure of beam structure with locally resonance units introduced. By changing the length of beam, natural frequency of locally resonance unit can be adjusted to the band gap of acoustic wave. Figure $3 \mathrm{c}$ shows the locally resonance unit formed by introducing the mass block into the perforated elastic material. The resonant frequency of the locally resonant unit can be changed by adjusting the mass of the mass block, so as to realize the sound insulation control of a specific band. The sound insulation effect is shown in Figure 3d.

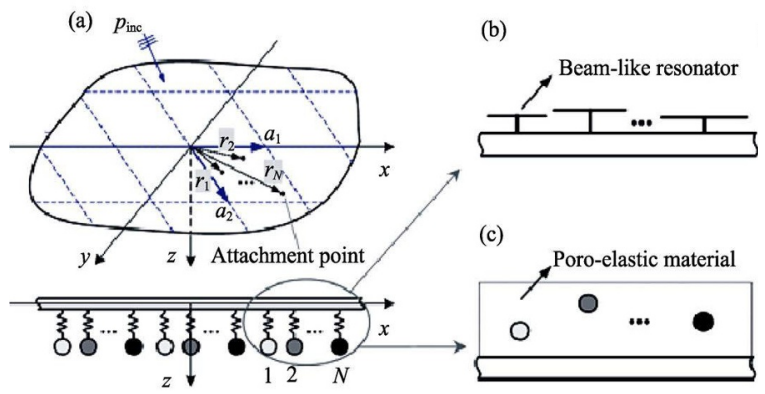

Fig. 3. Acoustic metamaterial prototype

\section{Locally resonance thin film type acoustic metamaterials}

\subsection{Locally resonance film-type acoustic metamaterial with additional mass vibrator}

Locally resonance film-type acoustic metamaterial with additional mass vibrator is composed of the elastic film of additional mass fixed in the supporting frame [6-7]. A mass - spring system composed of mass block and elastic film is used to selectively absorb elastic wave at a specific frequency. The typical structure of this material is shown in Figure 4.

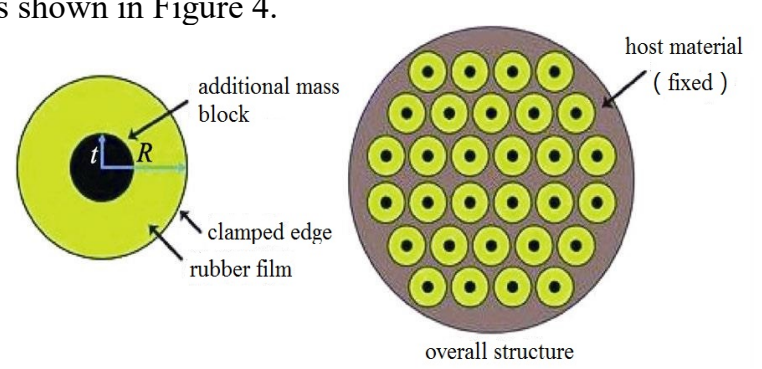

Fig. 4. Typical structure diagram

At present, the third-generation thin-film acoustic metamaterial structure has been successfully developed. Figures 5-7 are prototypes of the first to third-generation products [6-9]. This material can achieve isolation of sound waves in different bands by adjusting the tension of resonant film, the quality and position of additional mass, etc., and it can also achieve broadband sound insulation by layering[10-14].

The third-generation thin-film acoustic metamaterial structure was proposed in 2015. Metal electrode is introduced as an additional mass vibrator into locally resonance film[15-16]. Acoustic wave band is controlled by active phase modulation. This opens up new ideas for the active control of sound waves by locally resonance acoustic metamaterials[17-18].

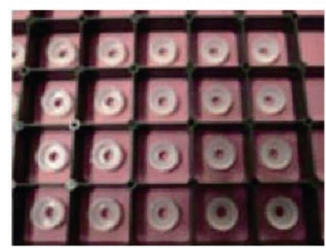

Fig. 5. The first-generation thin film acoustic metamaterial structure 

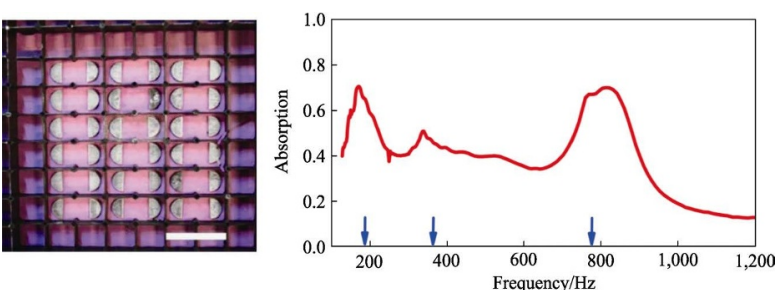

Fig. 6. The second-generation thin film acoustic metamaterial structure and sound insulation curve
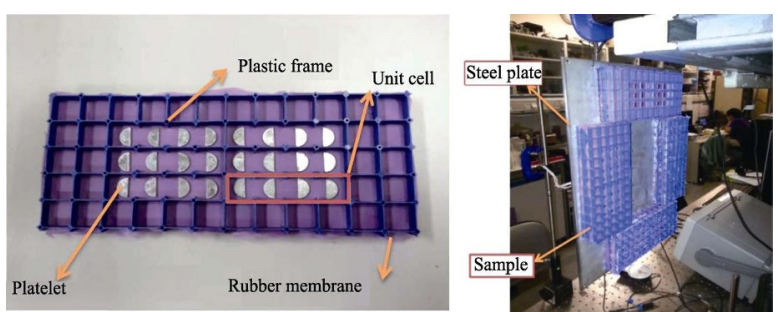

Fig. 7. The third-generation thin-film acoustic metamaterial sheet products and test site picture

\subsection{Thin-film acoustic metamaterial with no attached mass oscillator locally resonance}

Naify et al. introduced several layers of nylon film with a thickness of $7.7 \mu \mathrm{m}$ and a hole of $\varphi 10 \mu \mathrm{m}$ in the center into the aramide honeycomb core. The composite honeycomb core material was then combined with glass fiber reinforced epoxy resin panel with a thickness of 1 $\mathrm{mm}$ to form a honeycomb sandwich structure [19]. It was tested that sound transmission loss was increased by $2 \mathrm{~dB}$ and $4 \mathrm{~dB}$, and sound absorption coefficient was increased by 2.5 times and 1.5 times in the frequency range from $50 \mathrm{~Hz}$ to $1600 \mathrm{~Hz}$ after the installation of 1 and 2 layer of film, compared with that without nylon film. The schematic diagram of structure and its sound insulation curve are shown in Figure 8.
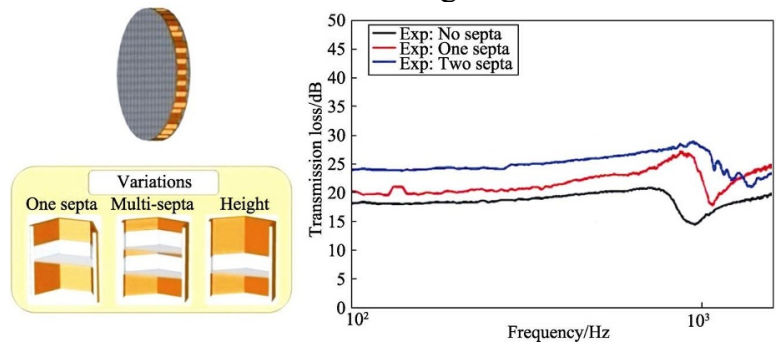

Fig. 8. Resonant sound absorption structure and sound insulation curve of honeycomb film

Researchers from North Carolina State University and the Massachusetts Institute of Technology directly compounded a rubber film with a thickness of about 0.25 $\mathrm{mm}$ and a honeycomb core to form a honeycomb sandwich structure. Similarly, without introducing a mass, the sound transmission loss and sound absorption coefficient are obtained Improved [20]. Compared with honeycomb sandwich structure without locally resonance film, the average sound transmission loss is increased from $31 \mathrm{~dB}$ to $40 \mathrm{~dB}$ in the frequency range of $50 \mathrm{~Hz} \sim 1600 \mathrm{~Hz}$. In the low frequency band below 500 $\mathrm{Hz}$, the average sound transmission loss is more than 50 $\mathrm{dB}$. The surface density of honeycomb sandwich structure with locally resonance film increases by about
$6 \%$, but the integrity of honeycomb sandwich structure is not affected. The schematic diagram of structure and its sound insulation curve are shown in Figure 9. The material has been used in the floors and ceilings of aircraft cabins.

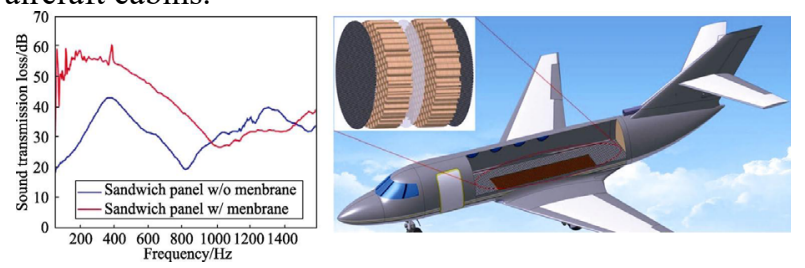

Fig. 9. Honeycomb film resonance structure, sound insulation curve and application case

\section{Conclusion}

Acoustic metamaterials have an acoustic forbidden band in the low frequency band, and have excellent acoustic performance in the forbidden band frequency range. Broadband sound absorption and sound insulation can be achieved by using multiple acoustic metamaterial structural units for combination or multilayer acoustic metamaterials for stacking combination. It has a broad application prospect in various equipment. Among them, thin-film acoustic metamaterial introduces locally resonance module into the traditional acoustic sandwich structure. Without affecting the integrity of the traditional sandwich structure and with a small increase in surface density, the sound absorption and insulation performance of the low frequency band can be greatly improved. It has been applied in foreign aviation products on a small scale.

\section{Acknowledgments}

This work was supported by the National Key Research and Development Program (No.2017YFC0307800).

\section{References}

1. S.H. Lee, C.M. Park, Y.M. Seo, Phys. Rev. Lett., 104, 543 (2010)

2. Z. Zhu, Y.Yang, N.Z. Zhang, Science, 289, 1734 (2000)

3. C.P. Chen, R.S. Lakers, Scripta Metallurgica et Materialia, 29, 395 (1993)

4. C.L. Ding, X.P. Zhao, Journal of Physics D: Applied Physics, 44, 215 (2011)

5. Y. Xiao, H.W. Ji, X. Wen, Journal of Sound and Vibration, 331, 5408 (2012)

6. Z. Yang, J. Mei, M.Yang, Physical Review Letters, 101, 204 (2008)

7. Z. Yang, H.M. Dai, N.H. Chan, Applied Physics Letters, 96, 906 (2010)

8. J. Mei, G.C. Ma, M. Yang, Nature Communications, 3, 756 (2012)

9. L. Sun, Y. Au, Y. Ka, AIP Advances, 6, 85 (2016) 
10. Z.M. Liu, Y. Pang, Acta. Mech. Sinica., 31, 45 (2015)

11. S. Zhang, C. Xia, N. Fang, Phys. Rev. Lett., 106, 124 (2011)

12. R.L. Harne, C.R. Fuller, J. Sound Vib., 331, 1859 (2012)

13. P. Wang, F. Casadei, S. Shan, Phys. Rev. Lett., 113, 1789 (2014)

14. C. Yan, H.L. Cheng, J. Pan, J. Acoust Soc. Am., 133, 201 (2013)

15. K. Song, S.H. Lee, K. Kim, Sci. Rep., 4, 4165 (2014)

16. N. Kaina, F. Lemoult, M. Fink, Nature, 525, 77 (2015)

17. H.H. Huang, C.T. Sun, New Journal of Physics, 11, 133 (2009)

18. T. Brunet, A. Merlin, B. Mascaro, Nat. Mater., 14, 384 (2015)

19. N. Christina, S. Matt, N. Steve, The Journal of the Acoustical Society of America, 8, 650 (2009)

20. N. Sui, X. Yan, T.Y. Huang, Applied Physics Letters, 106, 171 (2015) 